\title{
Determinación de arsénico y mercurio en agua superficial del lago de Atitlán
}

\author{
Francisco Pérez-Sabino ${ }^{1 *}$, Balmore Valladares-Jovel², Elisandra Hernández ${ }^{2}$, \\ Bessie Oliva², Marta Del Cid², Pedro Jayes-Reyes ${ }^{1}$ \\ ${ }^{1}$ Unidad de Análisis Instrumental y ²Departamento de Análisis Inorgánico, Facultad de Ciencias Químicas y Farmacia, \\ Universidad de San Carlos de Guatemala.
}

*Autor al que se dirige la correspondencia: fpsabino@yahoo.com

Recibido: 27 de mayo 2015 / Revisión: 23 de septiembre 2015 / Aceptado: 29 de octubre 2015 / Disponible en línea: 16 de noviembre 2015

\section{Resumen}

- 1 lago de Atitlán, considerado uno de los lagos más bellos del mundo, tiene una superficie de $125.7 \mathrm{~km}^{2}$, una - profundidad máxima de aproximadamente $350 \mathrm{~m}$, y se encuentra en el departamento de Sololá, en el occidente de Guatemala. Desde 2009 se han observado floraciones extensivas de cianobacterias en el lago, reflejando la contaminación del agua en la cuenca. Se determinaron los niveles de arsénico (As) y mercurio (Hg) en agua superficial del lago de Atitlán, por espectrofotometría de absorción atómica, en cuatro muestreos realizados en marzo, mayo, agosto y octubre de 2014 en 14 sitios. Diez sitios se encuentran localizados en el lago, habiendo sido definidos en estudios anteriores con base en su cercanía a poblaciones asentadas en la orillas del lago. Dos sitios corresponden a los principales ríos tributarios, y los restantes dos sitios a la entrada y salida de la planta de tratamiento Los Cebollales, ubicada en Panajachel. Los niveles de As encontrados en el lago de Atitlán, tributarios y en la planta de tratamiento, fueron superiores a $20 \mu \mathrm{g} / \mathrm{L}$. Los resultados indican que la contaminación del lago de Atitlán por As se debe principalmente a la geología de la cuenca, al localizarse en una zona volcánica. Los niveles cuantificables de As, son superiores al nivel máximo permitido para agua potable en la Norma de Agua Potable (NGO 29001) de la Comisión Guatemalteca de Normas (COGUANOR), por lo que el agua del lago de Atitlán no se considera apta para el consumo humano.

Palabras claves: Aguas residuales, espectrofotometría de absorción atómica, metales pesados, manejo sustentable

\begin{abstract}
L ake Atitlan is considered one of the most beautiful lakes in the world. It has $125.7 \mathrm{~km}^{2}$ and $350 \mathrm{~m}$ maximum depth. It is located at the western province Solola in the Guatemalan highlands. Since 2009 several cyanobacterial blooms have occurred at the lake, as consequence of the environmental degradation caused by water contamination into the basin. In this study pollution levels by arsenic (As) and mercury (Hg) in Lake Atitlan, were investigated by atomic absorption spectrophotometry. Four samplings were conducted in March, May, August and October 2014, collecting samples of water at 14 sampling sites, including the Wastewater Treatment Plant located at Panajachel. Presence of As above $20 \mu \mathrm{g} / \mathrm{L}$ was found in the water of Lake Atitlan, rivers and the wastewater treatment plant. The results indicate that contamination of Lake Atitlan regarding metals, is due majorly to the Basin geology located in a volcanic region. The levels of As are higher than recommended levels for drinking water in different regulations.
\end{abstract}

Keywords: Wastewater, atomic absorption spectrophotometry, heavy metals, sustainable management 


\section{Introducción}

Las aguas de ríos y lagos, al no existir contaminación antropogénica, generalmente presentan contenido de arsénico (As) menor que $10 \mu \mathrm{g} / \mathrm{L}$. En Brasil, en estudios realizados por el Centro Tecnológico de Saneamiento Básico (CETESB) en 1996, en el río Ribeiro de Iguape, Sao Paulo, se encontraron niveles elevados de As de 0.03 y $0.02 \mathrm{mg} / \mathrm{L}$ en aguas empozadas junto con desechos de minería de la Compañía Argentífera Furnas (CAF) y en los afluentes del río Furnas, respectivamente. En el monitoreo de las aguas brutas afluentes de las estaciones de tratamiento de aguas (ETAs) y aguas tratadas, analizadas en 1996, se encontraron concentraciones de As menores que $0.002 \mathrm{mg} / \mathrm{L}$ (CETESB, 1996).

La principal causa de intoxicación crónica por As inorgánico en el mundo, es la ingesta de agua de pozo contaminada. Se ha encontrado As en aguas subterráneas con niveles arriba del límite recomendado por la Organización Mundial de la Salud $(10 \mu \mathrm{g} / \mathrm{L})$ en varios países del mundo: India, China, Taiwan, Chile, Argentina, México y Estados Unidos (Chatterjjee \& Mukherjee, 1999; Chowdhury et al., 2000; Smith et al., 2000; Wyatt, Lopez, Olivas, \& Méndez, 1998).

Oliva, Pérez, Del Cid, Martínez y Valladares (2010) analizaron As en biomasa producto de la floración de cianobacterias ocurrida en octubre de 2009, encontrándose concentraciones de 2.013, 11.679 y 0.248 $\mu \mathrm{g} / \mathrm{g}$ en peso seco en muestras colectadas por arrastre con red de fitoplancton en los alrededores de San Buenaventura, San Pedro la Laguna y centro del lago, respectivamente, durante esta floración extensa, siendo los primeros sitios localizados en las cercanías de la orilla del lago. Además de estudios de agua subterránea de la cuenca en que se encontró As (Geólogos del Mundo, 2013), se consideraron como razones para investigar los niveles de As en agua del lago de Atitlán y sus afluentes. La contaminación por As puede tener también causas antropogénicas, como la fundición de minerales metálicos, herbicidas arsenicales y preservantes de madera, las cuales no son significativas en la cuenca del lago de Atitlán. En el mismo estudio, Oliva y colaboradores (2010) encontraron zinc $(\mathrm{Zn})$, plomo $(\mathrm{Pb})$, cadmio (Cd) y As en diferentes concentraciones promedio en las plantas acuáticas del lago, Potamogeton pectinatus (Zn: 11,000 mg/Kg; Pb: 156 mg/Kg; Cd: 120 mg/kg; As: $64 \mathrm{mg} / \mathrm{kg}$ ) Erigeria densa (Zn: $2513 \mathrm{mg} / \mathrm{kg}, \mathrm{Cd}: 42 \mathrm{~g} /$ kg; Pb: $91.5 \mathrm{mg} / \mathrm{kg}$; As: $115 \mathrm{mg} / \mathrm{kg}$ ), Eichornia crassipes (Zn: 3,210 mg/kg; Cd: 16 mg/kg; Pb: 151 mg/kg; As: 177 mg/kg), Typha dominguensis (Zn: 995 mg/kg; Cd: 5 $\mathrm{mg} / \mathrm{kg}$; Pb: $65.2 \mathrm{mg} / \mathrm{kg}$; As: $39.9 \mathrm{mg} / \mathrm{kg}$ ). También fueron encontrados metales en sedimentos del lago de Atitlán en concentraciones promedio para $\mathrm{Zn}(805 \mathrm{mg} / \mathrm{kg}), \mathrm{Cd}(185.5$ $\mathrm{mg} / \mathrm{kg}), \mathrm{Pb}(183.3 \mathrm{mg} / \mathrm{kg})$ no habiendo sido analizado el As. Concentraciones promedio de $\mathrm{Zn}(176 \mathrm{mg} / \mathrm{kg}$ ), $\mathrm{Pb}$ (36 $\mathrm{mg} / \mathrm{kg}$ ) y As (44 mg/kg) se han reportado en el lago de Amatitlán (Basterrechea, 1997), mientras que en el lago de Izabal, se han reportado concentraciones de $\mathrm{Zn}(86 \mathrm{mg}$ / $\mathrm{kg}$ ) y $\mathrm{Pb}(221 \mathrm{mg} / \mathrm{kg}$ ) no reportándose As (Basterrechea et al., 1993). De esta forma el Zn en dichos lagos se encontró en concentraciones menores a las encontradas en el lago de Atitlán en 2009, mientras que para el Pb se reportó una menor concentración promedio en el lago de Amatitán y mayor en el lago de Izabal, al compararse con las concentraciones en el lago de Atitlán.

Por otra parte, el mercurio $(\mathrm{Hg})$ es un contaminante tóxico, persistente y bioacumulativo (United Nations Enviroment Programme, 2013), el cual es biomagnificado en la red trófica, siendo uno de los metales pesados con mayor potencial tóxico. El Hg se encuentra en pequeña concentración en la naturaleza, sin embargo, los niveles del metal se han incrementado en las últimas décadas debido a emisiones provocadas por fuentes antropogénicas, como el uso de combustibles fósiles, la minería y fundición de metales y la quema de basura. En los ecosistemas acuáticos, el $\mathrm{Hg}$ se encuentra en forma metálica, como $\mathrm{Hg}$ iónico y formando compuestos organometálicos. El Hg inorgánico descargado en los ecosistemas acuáticos es incorporado principalmente en los sedimentos debido a su alta afinidad por el material particulado (Le Roux, Turner, Millward, Ebdon, \& Appriou, 2001). De esta forma, los sedimentos sirven como sumidero de $\mathrm{Hg}$, sin embargo, también representan una fuente de especies de $\mathrm{Hg}$ hacia la columna de agua, por la conversión del metal en compuestos organometálicos, principalmente metilmercurio, por medio de la acción de bacterias reductoras de sulfatos, las cuales se encuentran abundantemente en los sedimentos superficiales (Gilmour et al., 1998).

El metilmercurio es una potente neurotoxina, siendo muy estable y presentando la capacidad de penetrar las membranas de los seres vivos, representando un riesgo para la salud reproductiva y neurológica de peces, aves y mamíferos a bajas concentraciones (Scheuhammer, Meyer, Sandheinrich, \& Murray, 2007), al bioacumularse y biomagnificarse fácilmente (Mason, Laporte, \& Andres, 2000; Watras et al., 1998). El metilmercurio puede llegar así al ser humano a través del consumo de pescado contaminado, que es la principal ruta de exposición del ser humano al metilmercurio, pudiendo causarle problemas de salud como reducción 
del campo visual, disturbios de movilidad, deterioro mental, parálisis y muerte (Bisinoti \& Jardim, 2004).

Por lo anterior, se analizaron $\mathrm{As} \mathrm{y} \mathrm{Hg}$ en agua del lago de Atitlán, con el propósito de evaluar si sus concentraciones representan un riesgo para la población humana que consume agua del lago, como una herramienta para la toma de decisiones por autoridades ambientales de la cuenca del lago de Atitlán.

\section{Materiales y métodos}

\section{Área de estudio}

El lago de Atitlán se localiza en $14.7^{\circ}$ latitud $\mathrm{N}$ y $91.2^{\circ}$ longitud $\mathrm{O}$ en el departamento de Sololá, a $1,562 \mathrm{msnm}$ a una distancia de $145 \mathrm{~km}$ de la ciudad de Guatemala, en la región de la cadena volcánica que atraviesa Guatemala. El área total de la cuenca del lago de Atitlán es de $580 \mathrm{~km}^{2}$ y del espejo de agua del lago es de $125.7 \mathrm{~km}^{2}$, presentando una profundidad máxima de alrededor de $350 \mathrm{~m}$. El lago es de origen volcánico, presentando una forma ovalada de $21 \times 18 \mathrm{~km}$, con dos bahías que corren en dirección al sur, a los lados de los volcanes Atitlán y Tolimán (LaBastille, 1988). Los principales tributarios del lago de Atitlán, son el río Quiscab formado en el municipio de Sololá, a partir de la unión de los ríos Zarbabal y Novillero, con una longitud de $22.25 \mathrm{~km}$, drenando parte de las aguas residuales del municipio de Sololá; y el río San Francisco, también conocido como río Panajachel, que presenta una longitud de $15.6 \mathrm{~km}$ y desemboca en el lago, en la población de Panajachel.

\section{Toma de muestras}

Se seleccionaron 10 sitios de muestreo para la toma de muestras de agua para el análisis de metales pesados en el lago de Atitlán y sus dos principales tributarios, el río Quiscab y el río San Francisco, tomándose como base una investigación anterior sobre la contaminación del agua del lago (Oliva et al., 2010), además de dos sitios adicionales, para la colecta de aguas residuales, uno en la entrada y otro en la salida de la planta de tratamiento Los Cebollales, ubicada en Panajachel (Figura 1), teniendo 14 puntos en total. Se realizaron cuatro muestreos a lo largo del año por conveniencia, en los meses de marzo, mayo, agosto y octubre de 2014. En cada sitio se tomó una muestra de agua superficial de $1.0 \mathrm{~L}$ en botellas de polipropileno, a una profundidad de $30.0 \mathrm{~cm}$, enjuagando la botella tres veces con el agua del sitio de muestreo, antes de tomar la muestra.

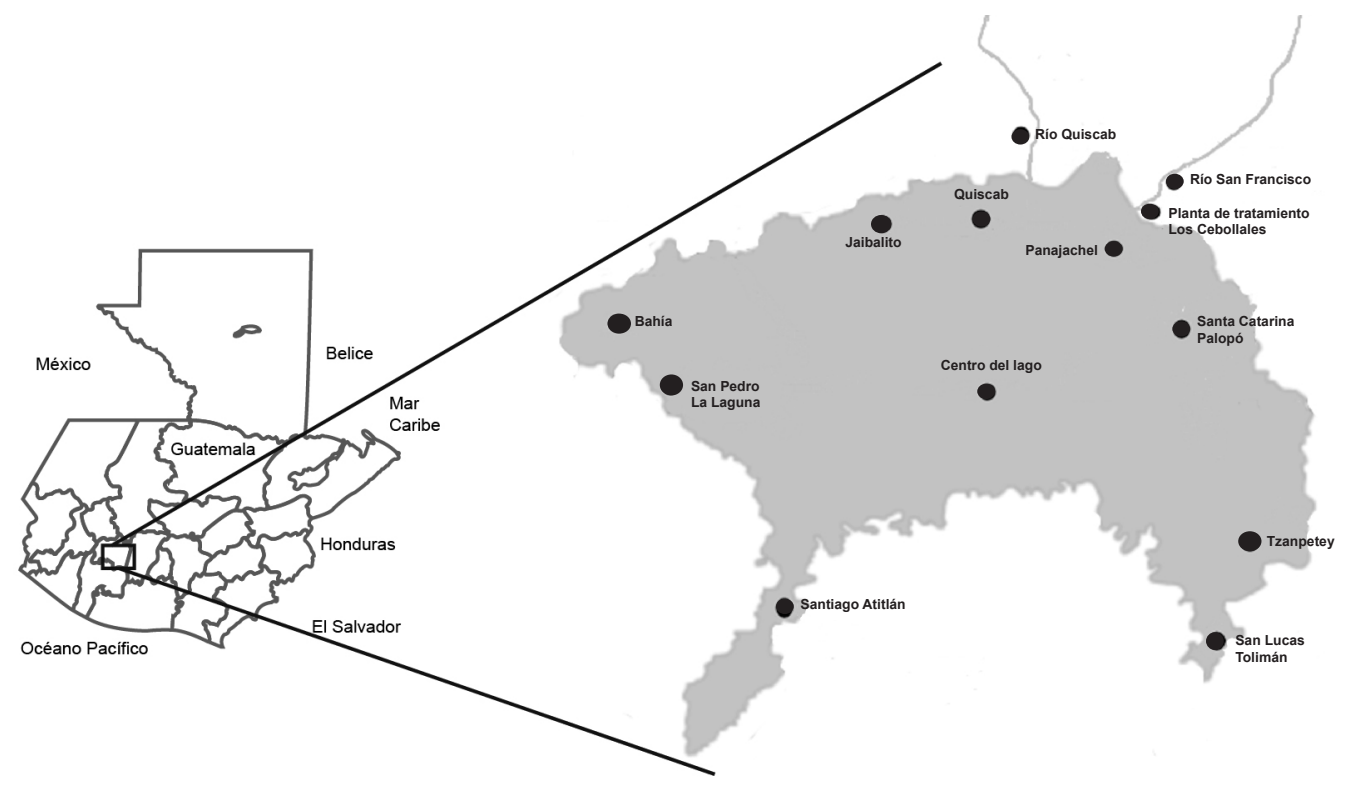

Figura 1. Mapa donde se ubican los puntos de muestreo en el Lago de Atitlán. 


\section{Análisis de las muestras}

Para la cuantificación del As y el Hg, se prepararon las muestras de agua y curvas de calibración en el Laboratorio de Investigación Química y Ambiental de la Escuela de Química de la Facultad de Ciencias Químicas y Farmacia de la Universidad de San Carlos de Guatemala, según métodos part 3114C y part 3112B para As y Hg respectivamente (American Public Health Association, 1998) por espectrofotometría de absorción atómica con generador de hidruros para As y con vapor frío para Hg (AAS, modelo S4 VP100, Thermo Fisher Scientific, application note AN40729 y application note AN40181_E 10/03C). Las curvas de calibración se prepararon utilizando estándares trazables al NIST. A cada muestra analizada por triplicado se le calculó el promedio y desviación estándar. La calidad de los resultados se asegura por medio de la medición de un estándar a cada cuatro lecturas de muestras.

\section{Resultados}

Se analizaron el As y el $\mathrm{Hg}$ en muestras de agua superficial colectadas en todos los sitios de muestreo en los cuatro meses, con excepción de los ríos San Francisco y Quiscab muestreados en tres de los cuatro meses. Los promedios y desviación estándar de los datos de As y Hg correspondientes a los análisis de cada muestra realizados por triplicado, se resumen en las Tablas 1 y 2.

En todos los puntos de muestreo se detectó As, encontrándose las concentraciones más bajas en el río Quiscab y las más altas en la entrada y salida de la planta de tratamiento de Panajachel. Así mismo el primer muestreo realizado en marzo tuvo las concentraciones más altas (Tabla 1). En cuanto a $\mathrm{Hg}$ en los dos primeros muestreos, marzo y mayo, en algunos puntos las concentraciones estuvieron por debajo del límite de detección. En agosto y octubre se encontraron niveles cuantificables en todos los sitios muestreados, siendo en el muestreo de agosto en el que se encontraron las concentraciones más altas de los cuatro muestreos realizados en el año (Tabla 2).

\section{Discusión}

Se considera que la presencia de As y Hg en la cuenca del lago de Atitlán es provocada por la geología de la cuenca, ya que no hay industria fuerte asentada en la misma. Se ha sugerido que el As en solución acuosa en la cuenca del lago de Atitlán se origina de

Tabla 1

Concentración promedio de arsénico en aguas del lago de Atitlán $(\mu g / L)$ con su desviación estándar

\begin{tabular}{lcccc}
\hline Sitio de muestreo & marzo 2014 & mayo 2014 & agosto 2014 & octubre 2014 \\
\hline Panajachel & $24.9 \pm 0.3$ & $17.1 \pm 0.2$ & $15.7 \pm 0.3$ & $17.5 \pm 0.1$ \\
Quiscab & $28.2 \pm 0.1$ & $17.3 \pm 0.1$ & $17.3 \pm 0.3$ & $13.0 \pm 0.3$ \\
Jaibalito & $29.7 \pm 0.1$ & $18.1 \pm 0.1$ & $06.4 \pm 0.2$ & $13.4 \pm 0.1$ \\
Centro Lago & $22.9 \pm 0.1$ & $18.6 \pm 0.1$ & $14.0 \pm 0.1$ & $14.5 \pm 0.2$ \\
Bahía & $29.7 \pm 0.1$ & $19.0 \pm 0.0$ & $14.6 \pm 0.2$ & $16.1 \pm 0.2$ \\
San Pedro & $27.8 \pm 0.1$ & $18.7 \pm 0.1$ & $15.1 \pm 0.1$ & $16.5 \pm 0.2$ \\
Santiago & $28.5 \pm 0.1$ & $18.7 \pm 0.0$ & $13.9 \pm 0.1$ & $16.5 \pm 0.1$ \\
San Lucas & $29.6 \pm 0.2$ & $19.2 \pm 0.1$ & $17.4 \pm 0.1$ & $18.2 \pm 0.1$ \\
Santa Catarina & $28.7 \pm 0.1$ & $19.9 \pm 0.1$ & $14.4 \pm 0.2$ & $18.2 \pm 0.2$ \\
Tzanpetey & $29.1 \pm 0.1$ & $19.6 \pm 0.1$ & $17.4 \pm 0.2$ & $18.4 \pm 0.1$ \\
Río San Francisco & $25.0 \pm 0.1$ & $17.5 \pm 0.1$ & $\mathrm{~N} \mathrm{M}$ & $09.0 \pm 0.3$ \\
Río Quiscab & $12.4 \pm 0.4$ & $07.7 \pm 0.1$ & $06.4 \pm 0.2$ & $\mathrm{~N} \mathrm{M}$ \\
Entrada Planta Tratamiento & $53.1 \pm 0.4$ & $33.3 \pm 0.1$ & $23.0 \pm 0.1$ & $36.8 \pm 0.5$ \\
Salida Planta Tratamiento & $107.0 \pm 0.3$ & $37.1 \pm 0.2$ & $41.1 \pm 0.5$ & $17.1 \pm 0.6$ \\
\hline
\end{tabular}

Nota. NM $=$ No Muestreado. 
Tabla 2

Concentración promedio de mercurio en aguas del lago de Atitlán $(\mu \mathrm{g} / \mathrm{L})$ con su desviación estándar

\begin{tabular}{lcccc}
\hline Sitio de muestreo & marzo 2014 & mayo 2014 & agosto 2014 & octubre 2014 \\
\hline Panajachel & DLD & DLD & $3.8 \pm 0.2$ & $4.3 \pm 0.1$ \\
Quiscab & DLD & DLD & $3.3 \pm 0.2$ & $2.5 \pm 0.7$ \\
Jaibalito & $1.1 \pm 0.0$ & DLD & $3.1 \pm 0.1$ & DLD \\
Centro Lago & $0.3 \pm 0.0$ & DLD & $2.6 \pm 0.2$ & $3.2 \pm 0.2$ \\
Bahía & $1.1 \pm 0.0$ & DLD & $2.8 \pm 0.2$ & $0.4 \pm 0.1$ \\
San Pedro & $0.9 \pm 0.0$ & $8.2 \pm 0.0$ & $2.9 \pm 0.2$ & $0.7 \pm 0.0$ \\
Santiago & DLD & $0.5 \pm 0.0$ & $2.9 \pm 0.2$ & $0.6 \pm 0.0$ \\
San Lucas & $0.4 \pm 0.0$ & $0.5 \pm 0.0$ & $2.7 \pm 0.1$ & DLD \\
Santa Catarina & DLD & $0.9 \pm 0.0$ & $2.8 \pm 0.2$ & $0.5 \pm 0.0$ \\
Tzanpetey & $0.6 \pm 0.0$ & DLD & $3.0 \pm 0.2$ & $0.2 \pm 0.0$ \\
Río San Francisco & $2.4 \pm 0.0$ & DLD & NM & $0.5 \pm 0.0$ \\
Río Quiscab & $1.9 \pm 0.0$ & $2.4 \pm 0.1$ & $3.5 \pm 0.1$ & NM \\
Entrada Planta Tratamiento & $1.2 \pm 0.0$ & DLD & $2.9 \pm 0.3$ & DLD \\
Salida Planta Tratamiento & $0.6 \pm 0.1$ & DLD & $2.8 \pm 0.1$ & $0.3 \pm 0.0$ \\
\hline
\end{tabular}

Nota. $\mathrm{NM}=$ No Muestreado, DLD = debajo del límite de detección.

depósitos piroclásticos asociados a antiguas erupciones freatomagmáticas o de rocas afectadas por alteración hidrotermal actual (Geólogos del Mundo, 2013)

En todas las muestras se han encontrado niveles detectables de As. En la planta de tratamiento fueron encontradas concentraciones más altas de As en la salida que en la entrada, habiéndose encontrado As arriba de $100 \mu \mathrm{g} / \mathrm{L}$ en marzo en la salida de la planta y niveles arriba de $20 \mu \mathrm{g} / \mathrm{L}$ en todos los sitios de muestreo en el lago, en el mismo mes, siendo el límite máximo permitido para el agua potable, según la norma COGUANOR NGO 29001:99, de $10 \mu \mathrm{g} / \mathrm{L}$. Se encontró también la presencia de $\mathrm{Hg}$ en casi todos los sitios de muestreo en concentraciones de hasta $3.81 \mu \mathrm{g} / \mathrm{L}$, niveles que no son tan elevados como los de As, sin embargo, el límite máximo permitido para agua potable, según la norma COGUANOR NGO 29001:99, es de $1 \mu \mathrm{g} / \mathrm{L}$, límite que fue superado en diferentes sitios de muestreo en diferentes meses, siendo destacable que en agosto dicho límite fue superado en todos los sitios de muestreo. Los resultados de As en cuatro sitios de muestreo representativos se presentan en la Figura 1 comparados con el valor guía de la norma.

Los resultados de presencia de As y $\mathrm{Hg}$ en el agua superficial del lago de Atitlán y sus afluentes, en concentraciones superiores a los límites recomendados por la norma de agua potable, son un hallazgo muy importante, ya que no se contaba con información sobre niveles de As en el lago, cuya agua es utilizada para consumo humano en varias comunidades. Además, existe una propuesta del proyecto Todos por el Lago, planteada en el Simposio de Investigaciones Científicas y Tecnológicas en la cuenca del lago de Atitlán, realizado en Panajachel en septiembre de 2014, de exportar las aguas residuales de la cuenca hacia la costa sur, para disminuir la descarga de nutrientes hacia el lago y lo que según los proponentes, trasladaría aguas fértiles a los cultivos de la costa sur. Sin embargo, de realizarse esta exportación de aguas residuales, se estarían contaminando los suelos y cultivos de la zona sur con metales de alta toxicidad en el mediano y largo plazo, por los volúmenes elevados que se esperaría se exportaran, siendo necesario también realizar estudios de retención de As por los suelos de la zona.

Para evaluar el riesgo para la salud humana de las concentraciones de As y $\mathrm{Hg}$, es necesario realizar la especiación de los mismos al existir estados de oxidación de ambos elementos que presentan mayor toxicidad. La especiación ha sido sugerida en estudios de contaminación de $\mathrm{Hg}$ en el sistema de lagunas Alvarado en México, 


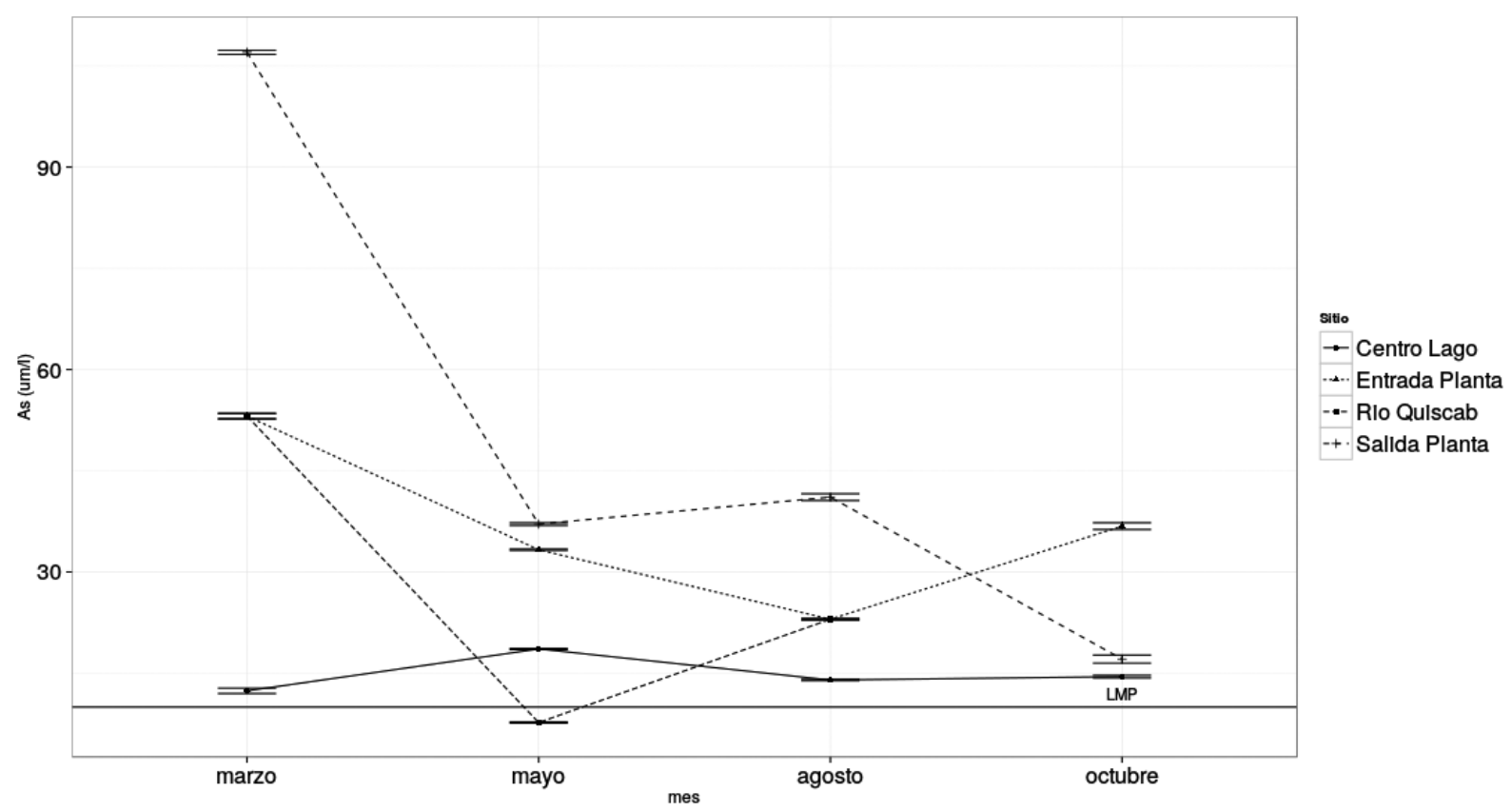

Figura 2. Concentraciones de As en agua de cuatro sitios de muestreo en la cuenca del lago de Atitlán, comparados con el nivel máximo permitido (LMP) para agua potable en la Norma COGUANOR NGO 29001:99.

en que se encontraron niveles de $\mathrm{Hg}$ en la columna de agua entre 1.0 y $12.7 \mu \mathrm{g} / \mathrm{L}$ (Guentzel, Portilla, Keith, $\&$ Keith, 2007), muy inferiores a las concentraciones de $\mathrm{Hg}$ encontradas en este estudio, y de especiación de As en el lago Colongulac, un lago endorreico localizado en Australia, en el cual se encontraron concentraciones de As total provocadas naturalmente, con una media de $61.4 \mu \mathrm{g} / \mathrm{L}$ en agua superficial y de $70.9 \mu \mathrm{g} / \mathrm{L}$ en la columna de agua (Madigan, Turoczy, \& Stagnitti, 2005).

En lagos que son contaminados fuertemente por actividades antrópicas, como el lago Titicaca, el $\mathrm{Hg}$ ha estado por debajo del límite de detección de $0.2 \mathrm{mg} / \mathrm{L}$ (Monroy, Maceda-Veiga, \& De Sostoa, 2014). En el lago Baiyangdian en China, se han encontrado concentraciones de ambos elementos en forma disuelta en los rangos de 1.1 y $22.1 \mathrm{ng} / \mathrm{L}$ en el caso del Hg, niveles inferiores a los encontrados en este estudio, y de 4,120 y 8,190 ng/L en el caso del As, niveles en el rango de las concentraciones de As encontradas en el lago de Atitlán; en dicha investigación se encontró que peces del lago Baiyangdian bioacumulan $\mathrm{As} \mathrm{y} \mathrm{Hg}$ a niveles que pueden presentar riesgo para la salud humana y la vida acuática (Chen, Pickhardt, Xu, \& Folt, 2008), por lo que es recomendable evaluar los niveles de dichos elementos en peces del lago de Atitlán.

Como principal conclusión, tanto el As como el $\mathrm{Hg}$ se encuentran en concentraciones cuantificables en el lago de Atitlán, encontrándose el As en concentraciones superiores a límites recomendados para agua potable, en la mayoría de sitios de muestreo. Por esta razón, el agua del lago no es apta para el consumo humano, y debe considerarse este aspecto en el análisis de la viabilidad de propuestas de exportación de aguas residuales hacia afuera de la cuenca.

\section{Agradecimientos}

El presente trabajo fue realizado con fondos (partida número 4.8.63.7.28) aportados por la Dirección General de Investigación (Digi) de la Universidad de San Carlos de Guatemala. Se agradece también la colaboración en la logística de los muestreos por parte de la Autoridad para el Manejo Sustentable de la Cuenca del Lago de Atitlán y su Entorno (AMSCLAE). 


\section{Referencias}

American Public Health Association, American Water Works Association, \& Water Environment Federation. (1998). Standard methods for the examination of water and wastewater (20th ed.). Washington, D.C.: Autor.

Basterrechea, M. (1997). El Lago de Amatitlán: Década de estudios limnológicos 1985 - 1995. Guatemala: Academia de Ciencias Médicas, Físicas y Naturales de Guatemala. .

Basterrechea, M., Solórzano, M., Juárez, Y., Palacios, R., Oliva, B., \& Aguilar E. (1993). Calidad del agua del Lago de Izabal y principales tributarios (Informe Técnico Final). Guatemala: DGENSEBV.

Bisinoti, M. C., \& Jardim, W. F. (2004). O comportamento do metilmercúrio (MetilHg) no ambiente. Química Nova, 27(4), 593-600.

Chatterjjee, A., \& Mukherjee, A. (1999). Hydrogeological investigation of ground water arsenic contamination in South Calcuta. Science of the Total Environment, 225(3), 249-262.

Centro Tecnológico de Saneamiento Básico. (1996). Avaliação da qualidade do Rio Ribeira de Iguape (Relatório Complementar). São Paulo, Brasil: Companhia de Tecnologia de Saneamento Ambiental.

Chen, C. Y., Pickhardt, P. C., Xu, M. Q., \& Folt, C. L. (2008). Mercury and arsenic ioaccumulation and eutrophication in Baiyangdian Lake, China. Water, Air and Soil Pollution, 190(1), 115-127. doi: 10.1007/s11270-007-9585-8

Chowdhury, U. K., Biswas, B. K., Chowdhury, T. R., Samanta, G., Mandal, B. K., Basu, G. C., ... Chakraborti, D. (2000). Groundwater arsenic contamination in Bangladesh and West Bengal, India. Environmental Health Perspectives, 108(5), 393-397.

Comisión Guatemalteca de Normas (2000). Norma Guatemalteca Obligatoria para el Agua Potable NGO 29.001.98.9

Geólogos del Mundo. (2013). Estudio hidrogeológico $y$ de recarga en la cuenca del Lago de Atitlán (Guatemala). Guatemala: Agencia Española de Cooperación Internacional para el Desarrollo.
Gilmour, C. C., Riedel, G. S., Ederington, M. C., Bell, J. T., Benoit, J. M., Gill, G. A., \& Stordal, M. C. (1998). Methylmercury concentrations and production rates across a trophic gradient in the northern Everglades. Biogeochemistry, 40(2), 327-345.

Guentzel, J. L., Portilla, E., Keith, K. M., \& Keith, E. O. (2007). Mercury transport and bioaccumulation in riverbank communities of the Alvarado Lagoon System, Veracruz State, Mexico. Science of the Total Environment, 388(1-3), 316-324. doi: 10.1016/j.scitotenv.2007.07.060

LaBastille, A. (1988). Lago de Atitlán. Nueva York: West of the Wind Publications.

Le Roux, S. M., Turner, A., Millward, G. E., Ebdon, L., \& Appriou, P. (2001). Partitioning of mercury onto suspended sediments in estuaries. Journal of Environmental Monitoring, 3(1), 37-42. doi: 10.1039/B007093I

Madigan, B. A., Turoczy, N., \& Stagnitti, F. (2005). Speciation of arsenic in a large endoheric lake. Bulletin of Environmental Contamination and Toxicology, 75, 1107-1114.

Mason, R. P., Laporte, J.-M., \& Andres, S. (2000). Factors controlling the bioaccumulation of mercury, methylmercury, arsenic, selenium, and cadmium by freshwater invertebrates and fish. Archives of Environmental Contamination and Toxicology, 38(3), 283-297.

Monroy, M., Maceda-Veiga, A., \& De Sostoa, A. (2014). Metal concentration in water, sediment and four fish species from Lake Titicaca reveals a large-scale environmental concern. Science of the Total Environment, 487, 233-244. doi: 10.1016/j. scitotenv.2014.03.134

Oliva, B., Pérez, J. F., Del Cid, B., Martínez F. J., \& Valladares, B. (2010). Estudio de contaminantes ecotóxicos en agua y organismos acuáticos del Lago de Atitlán (Inf-2009-064). Guatemala: Universidad de San Carlos de Guatemala, Dirección General de Investigación, Facultad de Ciencias Químicas y Farmacia.

Scheuhammer, A. M., Meyer, M. W., Sandheinrich, M. B., \& Murray, M. W. (2007). Effects of environmental methylmercury on the health of wild birds, mammals and fish. Ambio, 36(1), 12-18. 
Smith, A. H., Arroyo, A. P., Mazumder, D, N., Kosnett, D. N., Hernandez, A. L., Beeris, M., ... Moore, L. E. (2000). Arsenic-induced skin lesions among Atacameño people in Northern Chile despite good nutrition and centuries of exposure. Environmental Health Perspectives, 108(7), 617620.

United Nations Environment Programme. (2013). Global Mercury Assessment 2013: Sources, emissions, releases and environmental transport. Geneva, Switzerland: Autor.

Watras, C. J., Back, R. C., Halvorsen, S., Hudson, R. J., Morrison, K. A., \& Wente, S. P. (1998). Bioaccumulation of mercury in pelagic freshwater food webs. Science of the Total Environment, 219(2-3), 183-208. doi:10.1016/ S0048-9697(98)00228-9

Wyatt, C. J., Lopez, V., Olivasa, R. T., \& Méndez, R. O. (1998). Excretion of arsenic (As) in urine of children, 7-11 years, exposed to elevated levels of As in the City Water Supply in Hermosillo, Sonora, México. Environmental Research, 78(1), 19-24. doi:10.1006/enrs.1998.3844 